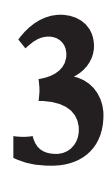

\title{
MODELO ESTRUCTURAL DE FACTORES EXTRÍNSECOS INFLUYENTES EN EL FLIPPED LEARNING
}

\section{(STRUCTURAL MODEL OF INFLUENTIAL EXTRINSIC FACTORS IN FLIPPED LEARNING)}

Santiago Mengual-Andrés

Universidad de Valencia

Jesús López Belmonte

Universidad Internacional de Valencia

Arturo Fuentes Cabrera

Santiago Pozo Sánchez

Universidad de Granada

DOI: $10.5944 / e d u c X X 1.23840$

\section{Cómo referenciar este artículo/How to reference this article:}

Mengual-Andrés, S.; López Belmonte, J.; Fuentes Cabrera, A., y Pozo Sánchez, S. (2020). Modelo estructural de factores extrínsecos influyentes en el flipped learning. Educación XX1, 23(1), 75-101, doi: 10.5944/educXX1.23840

Mengual-Andrés, S.; López Belmonte, J.; Fuentes Cabrera, A., \& Pozo Sánchez, S. (2020). Structural model of influential extrinsic factors in flipped learning. Educación XX1, 23(1), 75-101, doi: 10.5944/educXX1.23840

\section{RESUMEN}

Los avances tecnológicos han provocado que el ámbito de la educación se vea influenciado por la aparición de nuevos modelos de enseñanza y aprendizaje mediados por la gran cantidad de recursos digitales y dispositivos electrónicos que cuenta hoy el profesorado. Uno de los enfoques metodológicos que está emergiendo como consecuencia de la innovación educativa es el flipped learning. Este modelo de enseñanza y aprendizaje se sustenta en la idea de que los discentes puedan visualizar y trabajar los contenidos de las próximas sesiones presenciales en el aula fuera del entorno académico, con la finalidad de dedicar el mayor tiempo posible en clase a la resolución de problemas y al despliegue de un trabajo más práctico, participativo y activo. De tal modo, se consigue una mayor motivación 
que beneficia el proceso de aprendizaje. El objetivo de este estudio se ha centrado en conocer la incidencia de factores externos como el contexto familiar, la autonomía, la autoestima y la motivación del alumnado sobre el enfoque flipped learning. Para ello se ha desarrollado un método cuantitativo a través de un diseño experimental de corte longitudinal y de naturaleza descriptiva y correlacional. Se ha tomado una muestra diacrónica (20132018) de 607 discentes de la Facultad de Educación, Economía y Tecnología de Ceuta (España). Para la recogida de datos se ha empleado un cuestionario ad hoc previamente validado. Los resultados revelan que los estudiantes que cuentan con un contexto familiar idóneo, además de adecuados valores en la autonomía, motivación y autoestima alcanzan óptimos valores en los resultados de aprendizaje. Asimismo, los discentes de mayor edad obtienen mejores valoraciones sobre los mismos. En cambio, los que disponen de obligaciones laborales reflejan un descenso de estos, siendo el sexo de los participantes no determinante en los resultados de aprendizaje.

\section{PALABRAS CLAVE}

Tecnologías de la información y comunicación; innovación pedagógica; proceso de aprendizaje; flipped learning; condiciones de aprendizaje; análisis estructural.

\section{ABSTRACT}

Technological advances have caused the field of education to be influenced by the emergence of new teaching and learning models mediated by the large amount of digital resources and electronic devices that teachers have today. One of the methodological approaches that is emerging as a consequence of educational innovation is flipped learning. This model of teaching and learning is based on the idea that students can visualize and work on the contents of the next classroom sessions outside the academic environment, in order to spend as much time as possible in class solving problems and the deployment of more practical, participatory and active work. In this way, greater motivation is achieved that benefits the learning process. The objective of this study has been to discover the incidence of external factors such as family context, autonomy, self-esteem and the motivation of students on the flipped learning approach. For this, a quantitative method has been developed through an experimental design of longitudinal cut and of descriptive and correlational nature. A diachronic sample (2013-2018) of 607 students was taken from the Faculty of Education, Economy and Technology of Ceuta (Spain). A previously validated ad hoc questionnaire was used to collect data. The results reveal that students who 
have a suitable family context, as well as adequate values in autonomy, motivation and self-esteem achieve optimal values in learning outcomes. Likewise, older students obtain better ratings. On the other hand, those with labor obligations reflect a decrease in the same, with the sex of the participants not determining the learning outcomes.

\section{KEY WORDS}

Information and communication technologies; educational innovation; learning process; flipped learning; learning conditions; structural analysis.

\section{INTRODUCCIÓN}

Los continuos cambios acaecidos durante la última década en aspectos cruciales a nivel poblacional, económico o tecnológico han contribuido considerablemente a la modificación del ámbito educativo actual (Rotellar y Cain, 2016). Se ha producido una modificación en la forma con la que se accede a la información y al conocimiento en este nuevo panorama social (López y Bernal, 2019) conectado por la tecnología (Barbosa y Amariles, 2019), tornándose ineludible una modificación de los procesos de enseñanza y aprendizaje para que avancen hacia una realidad centrada en las tecnologías de la información y la comunicación (en adelante, TIC) y en la exploración de entornos virtuales de aprendizaje (McCarthy, 2016). En este sentido, la tecnología educativa se ha convertido en todo un apoyo a la docencia, mejorando la flexibilidad del aprendizaje y otorgando nuevas experiencias y posibilidades educativas (Cendon, 2018).

Durante los últimos años se ha incrementado la apuesta estatal a favor de la inclusión de las TIC con fines didácticos en los centros educativos, centros que a su vez están realizando un gran esfuerzo por poner en práctica -dentro de sus proyectos pedagógicos- medidas formativas innovadoras complementadas con la tecnología. En la misma línea, las universidades están asumiendo e integrando las TIC en los procesos formativos con la intención de dar respuesta a los retos de aprendizaje de una era digital (Cabero, Arancibia, y Del Prete, 2019). En esta tendencia hacia la optimización de los procesos instructivos resulta fundamental la existencia de un rol activo del discente para que pueda alcanzar con éxito los objetivos formulados para su proceso de aprendizaje y que -de esta forma- pueda percibirse por sus usuarios como una formación útil y eficaz (Jovanović, Gašević, Dawson, Pardo, y Mirriahi, 2017). Asimismo, la proliferación de las 
TIC ha supuesto que el profesorado asuma nuevos roles y competencias en su labor profesional (Guri-Rosenblit, 2018).

Bajo este panorama formativo, resulta pertinente destacar el gran número de enfoques pedagógicos que durante los últimos años han proliferado profusamente en el marco educacional. Si bien en muchos aspectos presentan multitud de concomitancias, su efectividad es a día de hoy una cuestión que provoca un importante debate investigativo. Dentro de dicha amalgama metodológica, nos encontramos con un enfoque que recientemente ha adquirido un protagonismo capital: el flipped learning (Sánchez, 2017; Zainuddin, Habiburrahim, Muluk, \& Keumala, 2019). Esta modalidad formativa de naturaleza mixta (Lee, Lim, \& Kim, 2017) ha llamado la atención de la comunidad educativa por su potencialidad y efectividad pedagógica (He, Holton, Farkas, \& Warschauer, 2016), en un intento de alejarse de las fórmulas academicistas que marginan aquellos aprendizajes adquiridos en ambientes informales o dentro del tiempo libre del discente mientras utilizan dispositivos digitales (Pereira, Fillol, y Moura, 2019).

De esta forma, el flipped learning se define como un enfoque pedagógico en el que se invierten los roles tradicionales del aula para que el alumnado inicie su aprendizaje fuera del aula tradicional y lo continúe, refuerce y complemente en su horario lectivo habitual (Llong, Cummins, \& Waugh, 2017). De esta forma, se produce un aprendizaje eminentemente práctico fundamentado en lo tecnológico (Froehlich, 2018) que permite una mayor flexibilización del tiempo y del espacio que otros enfoques formativos tradicionales (Boelens, Voet, \& Wever, 2018). Por su parte, El Miedany (2019) afirma que el aprendizaje invertido permite un mayor aprovechamiento del tiempo empleado en el aula para que el docente profundice en los contenidos de aprendizaje y el discente construya su propio conocimiento. En una línea similar, MacLeod, Yang, Zhu, \& Shi (2017) consideran que esa construcción del conocimiento por parte del alumno implica que se produzca una mayor interrelación con su grupo de iguales y se fomenten -de esta forma-medidas socializadoras con un importante índice de éxito a nivel motivacional.

Adentrándonos de forma pormenorizada en los elementos que mayor incidencia tienen dentro de la aplicación del enfoque flipped learning, resulta necesario destacar el papel de la motivación discente dentro de esta modalidad de aprendizaje. El aula invertida contribuye de forma óptima a que los discentes desarrollen una actitud positiva hacia el proceso de enseñanza (Lee, Park, \& Davis, 2018), produciendo altos niveles de motivación entre el alumnado (Tse, Choi, \& Tang, 2019), especialmente entre aquellos estudiantes habituados a un modelo de enseñanza tradicional (Huan, 2016). En una línea similar, en las investigaciones realizadas por Thai, De Wever, \& 
Valcke (2017) se constató que la motivación y el rendimiento del alumnado -dentro de un enfoque flipped learning- son superiores que en otros tipos de propuestas pedagógicas de similares características a nivel tecnológico, tales como el e-learning y el blended learning. De esta forma, el aula invertida se posiciona como un enfoque con un alto grado de eficacia con respecto a propuestas de corte más tradicional, pero también con respecto a otros enfoques pioneros que comparten características de su modelo instructivo (Sánchez, Jimeno, Pertegal, y Mora, 2019).

Por otra parte, la autorregulación del aprendizaje es un factor diferencial dentro de una asimilación significativa de los contenidos curriculares. El aula invertida contribuye a que el discente realice su propia planificación de los objetivos y a su eficacia volitiva (Chaves, Trujillo, y López, 2016), fomentando el papel activo del alumno, en tanto que se convierte en protagonista de su propia regulación del proceso de instrucción. De esta forma, las actividades cognitivas del alumnado dentro del flipped learning irán encaminadas hacia un aprendizaje autorregulado por el discente caracterizado por su tendencia a lo observacional y lo vicario (Cerezo, Bernardo, Esteban, Sánchez, y Tuero, 2015), requiriendo en el alumnado un alto grado de compromiso y voluntad positiva hacia la realización de ejercicios metacognitivos que impliquen habilidades de orden superior (Cabero y Llorente, 2015).

Conforme a lo evidenciado en investigaciones recientes, la autoestima del alumno se erige como un factor determinante para alcanzar el éxito formativo, y se encuentra íntimamente ligada a la calidad de su formación (Esquivel, 2018) y al nivel de consecución de los objetivos curriculares (Cabanach, Souto, Freire, y Ferradás, 2015). Por consiguiente, el aula invertida cobra especial importancia para afrontar esta tipología de cuestiones, en tanto que permite optimizar la experiencia instructora del alumnado, facilitando que consigan alcanzar los objetivos de aprendizaje (Awidi \& Paynter, 2019; Nortvig, Petersen, \& Hattesen, 2018), y experimenten una mejora significativa en las calificaciones obtenidas en las pruebas de evaluación (Karabulut, Jaramillo, \& Hassall, 2018).

Continuando el abordaje de la incidencia del flipped learning en el alumnado, Tourón y Santiago (2015) han constatado que el hecho de trabajar con material online permite un alto grado de flexibilidad dentro del aula ordinaria que contribuye -a su vez- a una mayor participación del alumno en comparación con el enfoque de aprendizaje tradicional, hecho que incide en el trabajo colaborativo por parte del grupo de discentes, tanto en la clase ordinaria como fuera del entorno escolar (Long, Logan, \& Waugh, 2016). Se produce -de esta forma- un incremento de la actividad cooperativa en aquellos espacios de aprendizaje enfocados hacia la inversión 
del aula tradicional (Kwon \& Woo, 2017), en una instrucción ubicua en la que la colaboración entre los participantes se potencia y complementa tanto dentro del aula como fuera de ella (Báez y Clunie, 2019).

Asimismo, esta configuración instructiva colaborativa propicia que el discente se enfrente a las dificultades propias del proceso formativo como ente protagonista, contribuyendo de esta forma a una potenciación de su capacidad para resolver problemas que se plantean de forma recurrente en la cotidianeidad del aula (Long et al., 2016). No obstante, varios estudios han comprobado que el modelo flipped learning conlleva a que el alumnado se enfrente a la resolución de problemas que no es capaz de solventar (Bognar, Sabli凶, \& Škugor, 2019), especialmente en lo referente a la adaptabilidad en el manejo de ciertas plataformas digitales (Yilmaz, 2017) y cuando el concepto a asimilar requiere de un proceso significativo de abstracción cognitiva (Hwang \& Lai, 2017). Por ende, será necesario comprender las particularidades del modelo invertido para poder aplicarlo en aquellas situaciones en las que su eficacia se verá potenciada, propiciando un entorno de aprendizaje en el que la resolución de problemas por parte del alumnado se encuentre respaldada por una formación específica previa que pueda suplir las carencias a nivel conceptual y experiencial del desempeño discente.

Por último, resulta prioritario reseñar que el contexto familiar tiene una importante incidencia dentro del desarrollo del proceso de enseñanza y aprendizaje en general, y de una óptima implantación del enfoque flipped learning en particular. Un contexto social problemático se caracteriza generalmente por situaciones precarias a nivel socioeconómico y por altos niveles de inconsistencia familiar, hechos que provocan en el discente una situación de inestabilidad afectiva perjudicial para su adaptación al aula que repercute de manera ineludible en su rendimiento académico (Tique et al., 2018). Por ello, gran parte del éxito de la implantación de esta modalidad formativa dependerá de la puesta en práctica de un estudio por parte del docente para determinar las particularidades del contexto familiar de su alumnado, y así poder individualizar y adaptar el modelo de aula invertida a las características de cada discente, contribuyendo hacia una óptima aclimatación del educando, con especial atención a aquel que vivencia dificultades de diversa índole dentro de su contexto familiar (Medranda y Romero, 2018).

Una revisión sistemática sobre el estado de la cuestión revela que el flipped learning aún no ha alcanzado un pleno desarrollo investigativo en comparación con otras metodologías emergentes. A pesar de ello, la literatura sobre este arte se ha centrado en medir su eficacia con respecto a metodologías de corte tradicional, así como verificar los beneficios 
alcanzados en diversos indicadores académicos tras su aplicación didáctica, tales como la motivación, el trabajo colaborativo, la autorregulación y los resultados de aprendizaje, entre los aspectos más destacados (Hinojo, Aznar, Romero, \& Marín, 2019).

En el estudio que se presenta, con el propósito de buscar un nuevo punto de inflexión con respecto a investigaciones anteriores, se ha tomado en consideración -además de la motivación- otro tipo de factores que pueden causar incidencia en los resultados de aprendizaje obtenidos por los discentes en el uso de tal enfoque innovador, como son su contexto familiar, su autoestima y su autonomía, así como averiguar cuál ejerce mayor determinación.

Por tanto, el objetivo de este estudio se focaliza en conocer la influencia de factores externos y constitutivos a la metodología flipped learning sobre los resultados de aprendizaje obtenidos por el discente en el proceso de enseñanza y aprendizaje. Del mismo modo, para valorar los resultados de aprendizaje anteriormente mencionados se han tomado diferentes aspectos como la participación, el trabajo colaborativo, la resolución de problemas y la calificación obtenida por el estudiantado.

De este objetivo general derivan los siguientes objetivos con mayor grado de especificidad:

a) Determinar la influencia del sexo de los participantes sobre los resultados de aprendizaje.

b) Comprobar el efecto de la edad de los estudiantes en los resultados de aprendizaje.

c) Averiguar la determinación del estado laboral de los sujetos sobre los resultados de aprendizaje.

\section{METODOLOGÍA}

El presente trabajo se ha desarrollado a través de un enfoque metodológico cuantitativo, enmarcándose en un diseño experimental de tipo descriptivo y correlacional de corte longitudinal.

\section{MUESTRA}

En el estudio han participado un total de 607 estudiantes universitarios del Grado de Educación Primaria de la Facultad de Educación, Economía y Tecnología de la Ciudad Autónoma de Ceuta (España), perteneciente a la Universidad de Granada (España). Para la selección de estos sujetos se 
ha seguido un muestreo por conveniencia dada la facilidad de acceso a los discentes y la disponibilidad de estos para desarrollar la investigación. Como se visualiza en la tabla 1 , se trata de una muestra diacrónica tomada en diferentes cursos académicos.

Tabla 1

Participantes del estudio

\begin{tabular}{cccccc}
\hline $\begin{array}{c}\text { Curso } \\
\text { académico }\end{array}$ & $2013-2014$ & $2014-2015$ & $2015-2016$ & $2016-2017$ & $2017-2018$ \\
\hline Alumnos & 126 & 121 & 134 & 117 & 109 \\
\hline
\end{tabular}

Fuente: Elaboración propia

La muestra resultante queda configurada por 381 mujeres $(62,76 \%)$ y 226 hombres $(37,23 \%)$, cuyas edades se encuentran en un intervalo entre 18 y 24 años $(M=21 ; D T=2,05)$. Asimismo, una de las variables sociales que se ha tenido en cuenta es la situación laboral, en la que un 35,09\% $(n=213)$ ha manifestado que dispone de un trabajo mientras cursa los estudios universitarios.

\section{INSTRUMENTO}

Para efectuar el proceso de recogida de datos se ha utilizado un cuestionario ad hoc derivado de otros instrumentos validados (ESFA, APM, AF-5 y ABAS-II) en población adulta y española, tomando los ítems más significativos para cumplir con los requerimientos de este estudio.

La razón para diseñar un cuestionario específico se sustenta en garantizar la objetividad de los datos y preservar la actitud favorable de los participantes ya que la utilización de los distintos instrumentos anteriormente citados daría lugar a que los estudiantes tuvieran que cumplimentar numerosos ítems, dificultando este proceso tan relevante en el proceso investigador.

Concretamente, el instrumento diseñado consta de 46 cuestiones estructurados en 5 dimensiones: 1-Sociodemográfica (SD)=6 ítems; 2-Motivación $(\mathrm{M})=12$ ítems; 3-Contexto familiar $(\mathrm{CF})=10$ ítems; 4-Autoestima $(\mathrm{AUTOE})=10$ ítems; 5-Autonomía $(\mathrm{AUTON})=8$ ítems .

Las cuestiones presentadas siguen un formato de respuesta variado, siendo en su mayoría de tipo Likert. Su escala se establece con base en una gradación del acuerdo del encuestado, donde 1 corresponde al valor "Nada" 
SANTIAGO MENGUAL-ANDRÉS, JESÚS LÓPEZ BELMONTE, ARTURO FUENTES CABRERA, SANTIAGO POZO SÁNCHEZ

MODELO ESTRUCTURAL DE FACTORES EXTRÍNSECOS INFLUYENTES EN EL FLIPPED LEARNING

y 5 al valor "Totalmente”. Otros ítems -singularmente de la dimensión SDse encuentran configurados mediante respuestas de elección cerrada.

El cuestionario fue sometido a un proceso de validación cualitativa por juicio de expertos, compuesto por catorce Doctores (ocho especialistas en tecnología educativa y seis especialistas en psicología social) con afiliación a diversas universidades españolas (Universidad de Granada, Universidad de Sevilla, Universidad de Extremadura, Universidad de Córdoba, Universidad de Málaga, Universidad de Alicante, Universidad Pablo de Olavide y Universidad Nacional de Educación a Distancia). La opinión vertida por estos expertos fue bastante positiva. De igual forma, para conocer el nivel de concordancia entre los juicios emitidos se aplicó el coeficiente Kappa de Fleiss, alcanzándose un resultado pertinente $(\mathrm{K}=0,874)$. Además, para determinar la fuerza de concordancia se halló la W de Kendall, obteniendo un valor elevado en la prueba $(\mathrm{W}=0,886)$. Antes de pasar a la validación estadística, se llevaron a cabo todas las orientaciones proporcionadas en el feedback de los expertos.

A continuación, siguiendo el método de Componentes Principales se efectuó un análisis factorial exploratorio con rotación Varimax (Tabla 2) en una muestra (316 discentes universitarios) independiente a los sujetos presentados en esta investigación, obteniéndose 4 factores con eigenvalue > 1 , siendo el criterio de asignación del ítem al factor que tuviera una carga factorial superior a 0,3 , revelado por el $65,11 \%$ de la varianza total. Como principio para valorar la viabilidad del análisis factorial se tomó el elevado número de correlaciones de la matriz $(89,6 \%)$ que presentaron un valor por encima de 0,3 , con un determinante inferior a 0,002. Por consiguiente, se efectuó el test de esfericidad de Bartlett reflejando una dependencia de las variables (Prueba Bartlett $=3.337,86 ; p<0,001$ ).

Por otro lado, la adecuación muestral se determinó por el test de Kaiser-Meyer-Olkin (KMO=0,933), reflejando que las correlaciones entre pares de variables son esclarecidas por el resto de variables, así como por las comunalidades $\left(\mathrm{h}^{2}>0,65\right)$. Igualmente, el Measures of Sampling Adequacy (MSA) obtuvo valores superiores a 0,83 , revelando la pertinencia del análisis factorial de la matriz de correlaciones.

En cuanto a la consistencia interna de los distintos ítems -hallada mediante el estadístico alfa de Cronbach- se arrojó una alta fiabilidad del instrumento $(\alpha>0,8)$, en base a los preceptos de Hernández, Fernández, y Baptista (2016). 
Tabla 2

Resultados del análisis factorial exploratorio

\begin{tabular}{llllll}
\hline & \multicolumn{4}{c}{ Factor } \\
\cline { 2 - 5 } & 1 & 2 & 3 & 4 \\
\hline
\end{tabular}

M1: Independencia y preferencia hacia el criterio propio en la toma de decisiones.

M2: Preferencia por formar parte del grupo de iguales y conseguir la aceptación del resto.

M3: Interés por competir, ganar y dirigir a otros; prestigio, popularidad y admiración.

M4: Deseo por el mantenimiento de relaciones equitativas y por evitar la rivalidad y el abuso de 0,676 poder.

M5: Estimulación hacia la superación de retos y hacia el éxito y la excelencia.

M6: Preferencia hacia la dosificación de esfuerzos y hacia la consecución de metas sin 0,593 perjuicio del bienestar.

M7: Gestión de las calificaciones resultantes de las pruebas en las que se es evaluado.

M8: Regularidad del ánimo y la actitud hacia el estudio.

0,755

M9: Preferencia por lo novedoso, lo variado y por el descubrimiento de nuevas formas de actuar.

M10: Búsqueda de la estabilidad dentro del entorno, evitando los cambios repentinos y la 0,902 incertidumbre.

M11: Deseo de ayudar a los demás y de tener un impacto positivo en la sociedad.

M12: Protección de sí mismo y de sus bienes materiales.

CF 1: Oportunidades de aprendizaje y relacionales con parientes adultos significativos.

CF2: Organización doméstica y contribución al desarrollo de una competencia educativa.

CF3: Existencia y efectividad de pautas y normativas de cumplimiento en el hogar. 


\begin{tabular}{llllll}
\hline & \multicolumn{5}{c}{ Factor } \\
\cline { 2 - 5 } & 1 & 2 & 3 & 4 \\
\hline
\end{tabular}

CF4: Fortaleza y estabilidad de la estructura familiar.

CF5: Influencia de antecedentes psicosociales de los progenitores.

CF6: Seguridad económica y de las condiciones de vivienda.

CF7: Búsqueda de apoyo dentro de la red familiar.

CF8: Desarrollo familiar y resiliencia.

CF9: Oportunidades de participación e integración comunitaria.

CF 10: Influencia de la conflictividad en el contexto de desarrollo familiar.

AUTOE1: Percepción del individuo de la calidad del desempeño de su rol como estudiante.

AUTOE2: Percepción de la persona de la calidad del desempeño en tareas cotidianas.

AUTOE3: Percepción de la facilidad del individuo para mantener o ampliar su red social.

AUTOE4: Percepción del individuo de la calidad del desempeño en las relaciones sociales interpersonales.

AUTOE5: Percepción de la persona de su estado emocional general.

AUTOE6: Percepción del desempeño emocional del individuo en la socialización con personas situadas en una posición superior dentro de la jerarquía social.

AUTOE7: Percepción de la persona de su implicación, participación e integración en el medio familiar.

AUTOE 8: Percepción del individuo de su dominio del afecto y la confianza
0,83

0,758

0,825

0,833

0,811

0,705

0,651

0,665

0,592

0,834

0,697

0,706

0,692

0,798

0,605 


\begin{tabular}{llllll}
\hline & \multicolumn{5}{c}{ Factor } \\
\cline { 2 - 5 } & 1 & 2 & 3 & 4 \\
\hline
\end{tabular}

AUTOE9: Percepción de la persona de la calidad de respuesta a situaciones específicas que conlleven el ejercicio del compromiso y la 0,749 implicación.

AUTOE10: Percepción del individuo de su aspecto físico y de su condición física.

0,825

AUTON1: Habilidades de habla y escucha necesarias para la comunicación.

0,647

AUTON2: Habilidades necesarias para su desenvolvimiento en la comunidad y para relacionarse con el resto.

AUTON3: Habilidades básicas de lectura, escritura, lógica y matemáticas necesarias para desenvolverse de forma independiente en 0,789 actividades cotidianas.

AUTON4: Habilidades necesarias para el cuidado básico del hogar y de otros lugares en los que el individuo realice sus actividades diarias.

AUTON5: Habilidades necesarias para el cuidado personal, de la salud y para hacer frente a enfermedades y lesiones.

AUTON6: Habilidades necesarias para planificar y participar en actividades de ocio.

AUTON7: Habilidades necesarias para la imposición de límites y la realización de elecciones.

AUTON8: Habilidades necesarias para el ejercicio de la independencia, el comportamiento responsable y el autocontrol.

\begin{tabular}{lrrrr}
\hline Autovalores & 3,55 & 2,36 & 1,19 & 1,04 \\
\% Varianza explicada & 21,98 & 19,53 & 13,71 & 9,89 \\
\% Varianza explicada acumulada & 21,98 & 41,51 & 55,22 & 65,11 \\
Alfa de Cronbach & 0,869 & 0,828 & 0,819 & 0,815 \\
\hline
\end{tabular}

Fuente: Elaboración propia 
SANTIAGO MENGUAL-ANDRÉS, JESÚS LÓPEZ BELMONTE, ARTURO FUENTES CABRERA, SANTIAGO POZO SÁNCHEZ

\section{PROCEDIMIENTO}

La investigación tuvo su origen en el año 2013 con la primera muestra de estudiantes matriculados en la asignatura de "Didáctica: Teoría y práctica de la enseñanza". Desde entonces y hasta la actualidad, se ha ido tomando una muestra discente de cada curso académico de dicha materia. El estudio presenta un análisis de los datos relativos a los últimos cinco años académicos.

La primera actuación llevada a cabo en cada curso fue obtener la colaboración del profesor encargado de impartir la asignatura mencionada, teniendo que adecuar la metodología de enseñanza y aprendizaje a los requerimientos de esta pesquisa, así como el consentimiento de los discentes para participar en el estudio, informándoles de que todos los datos recabados serán tratados con total anonimato.

Para el desarrollo de los contenidos de la asignatura, el docente aplicó una metodología flipped learning, apoyándose en un blog como espacio virtual para alojar los vídeos y -de tal manera- lograr la interacción del alumnado en entornos digitales. De esta forma, los estudiantes podían visualizar el material audiovisual (previos a la sesión) en cualquier momento y lugar fuera del entorno académico y formal.

Una vez impartidos todos los contenidos, el docente aplica su rúbrica evaluativa. En ella se recogen las valoraciones acerca de la participación en clase y en el blog (reportado mediante las analíticas de aprendizaje generadas), el trabajo colaborativo, la resolución de problemas (desde una perspectiva dicotómica) y la calificación obtenida en la prueba de evaluación de la asignatura. Dicha herramienta forma parte del seguimiento diario que el docente hace del desempeño de sus alumnos.

Finalmente, los investigadores aplican el cuestionario ad hoc validado para extraer toda la información posible sobre los factores externos que pueden interferir en los resultados de aprendizaje mediante el modelo flipped learning.

\section{ANÁLISIS DE DATOS}

Para el análisis estadístico descriptivo de la muestra se obtuvo el número de casos presentes en cada categoría y el porcentaje correspondiente para las variables cualitativas, así como la media y desviación típica para las variables cuantitativas. El análisis factorial exploratorio se efectuó para determinar la estructura factorial del cuestionario aplicado. Su viabilidad 
se evaluó con el test de esfericidad de Bartlett, el test de Kaiser-Meyer Olkin (KMO) y por los valores del Measures of Sampling Adequacy (MSA). Para llevar a cabo la factorización se utilizó el método de Componentes Principales con rotación Varimax, además del coeficiente Alfa de Cronbach para determinar la consistencia interna.

El análisis confirmatorio del modelo generado en el estudio exploratorio con ecuaciones estructurales se realizó mediante el método de extracción de máxima verosimilitud. De igual forma, el modelo de regresión lineal múltiple se ejecutó para determinar el efecto de las variables independientes consideradas en la predicción de los resultados de aprendizaje.

Todo el análisis estadístico desplegado se llevó a cabo con el programa IBM Statistical Package for the Social Sciences (SPSS) e IBM SPSS Amos v.25, tomando como diferencias consideradas estadísticamente significativas aquellas cuya $p<0,05$.

\section{RESULTADOS}

Como se visualiza en la figura 1, se confirma la adecuación del modelo obtenido en el análisis exploratorio, alcanzándose un modelo formado por 4 factores, con un total de 40 ítems. La estimación de los parámetros resultó estadísticamente significativa y las cargas factoriales se encontraron por encima de 0,58 , saturando - por consiguiente- cada una de las variables latentes por los indicadores. 


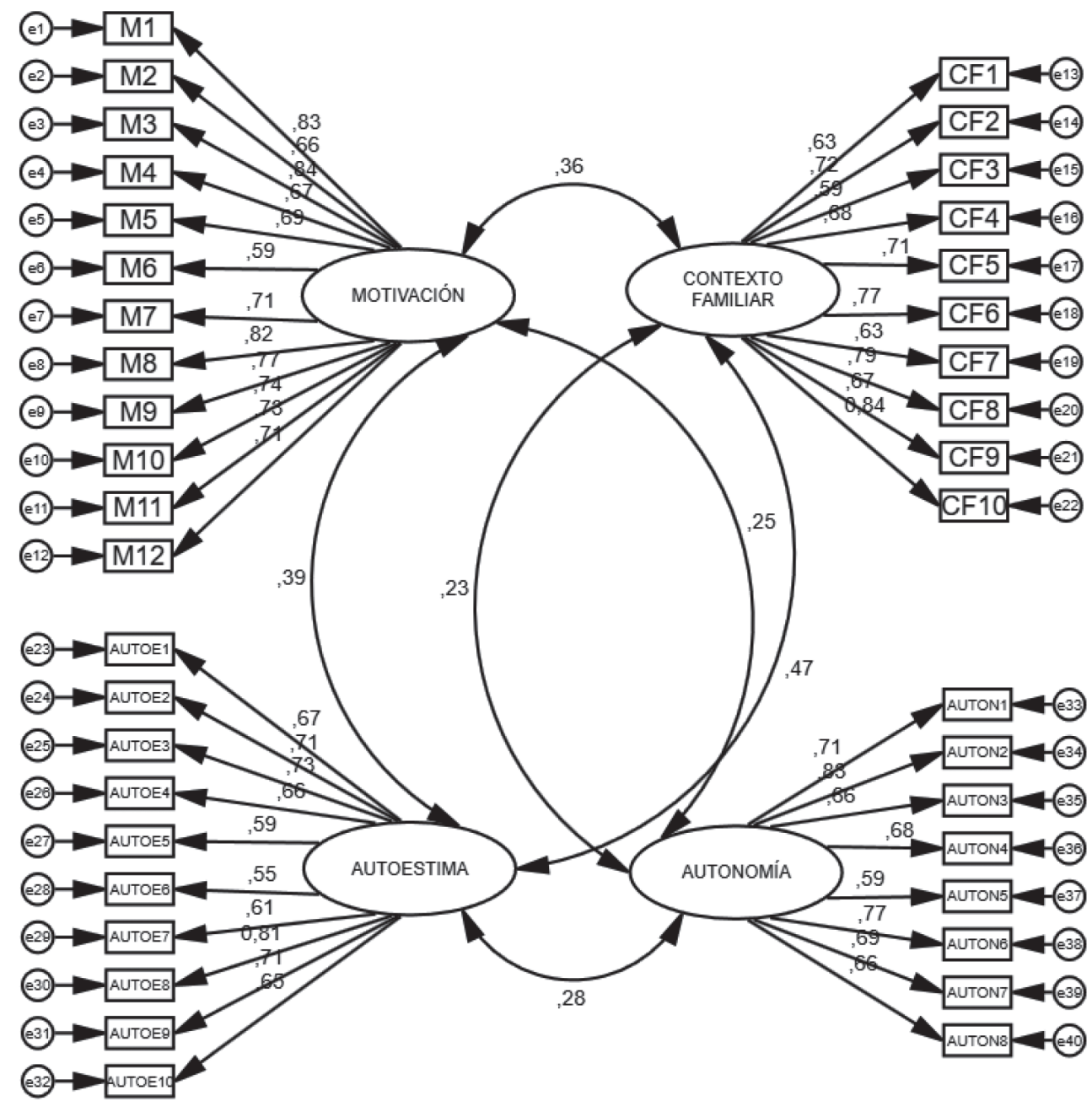

Figura 1. Ecuación estructural del AFC

Fuente: Elaboración propia

Como se muestra en la tabla 3, los índices de ajuste del modelo obtuvieron valores adecuados, afirmando la sustentabilidad del modelo presentado sobre la estructura factorial de la escala. En otro orden, se realizaron análisis en dos submuestras independientes y probabilísticas, cuyos resultados revelaron valores pertinentes. 
Tabla 3

Índices de bondad de ajuste del AFC

\section{$\chi 2 / g l \quad$ GFI AGFI CFI NFI TLI}

RMSEA (I.C. 90\%)

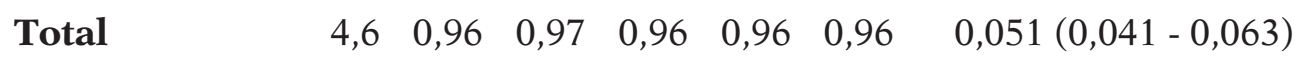

$\begin{array}{llllllll}\text { Submuestra } 1 & 3,41 & 0,95 & 0,95 & 0,96 & 0,95 & 0,96 & 0,053\end{array}(0,044-0,069)$

$\begin{array}{llllllll}\text { Submuestra } 2 & 3,22 & 0,95 & 0,96 & 0,95 & 0,96 & 0,95 & 0,052(0,043-0,071)\end{array}$

Fuente: Elaboración propia

La figura 2 representa el modelo final estimado para predecir los resultados de aprendizaje. Se aprecia que todos los parámetros resultaron estadísticamente significativos $(p<0,05)$ aunque con distinta influencia sobre tales resultados. El contexto familiar es la variable que presentó mayor efecto y de forma directa sobre los resultados de aprendizaje $(\gamma=0,63, p=0,013)$. Las siguientes variables, según su determinación, fueron la autonomía $(\gamma=0,42$, $p=0,021)$, motivación $(\gamma=0,21, p=0,037)$ y autoestima $(\gamma=0,13, p=0,044)$. El modelo permite explicar el $49 \%$ de la varianza de los resultados de aprendizaje. Sobre el ajuste del mismo (Tabla 4), los indicadores mostraron unos valores adecuados.

Tabla 4

Índices de bondad de ajuste del modelo final estimado

\begin{tabular}{lr}
\hline Indicador & Valor \\
\hline$\chi \mathbf{2} /$ gl & 2,11 \\
GFI & 0,97 \\
AGFI & 0,96 \\
CFI & 0,95 \\
NFI & 0,96 \\
TLI & 0,97 \\
RMSEA (I.C. 90\%) & $0,041(0,021-, 068)$ \\
\hline
\end{tabular}

Fuente: Elaboración propia 


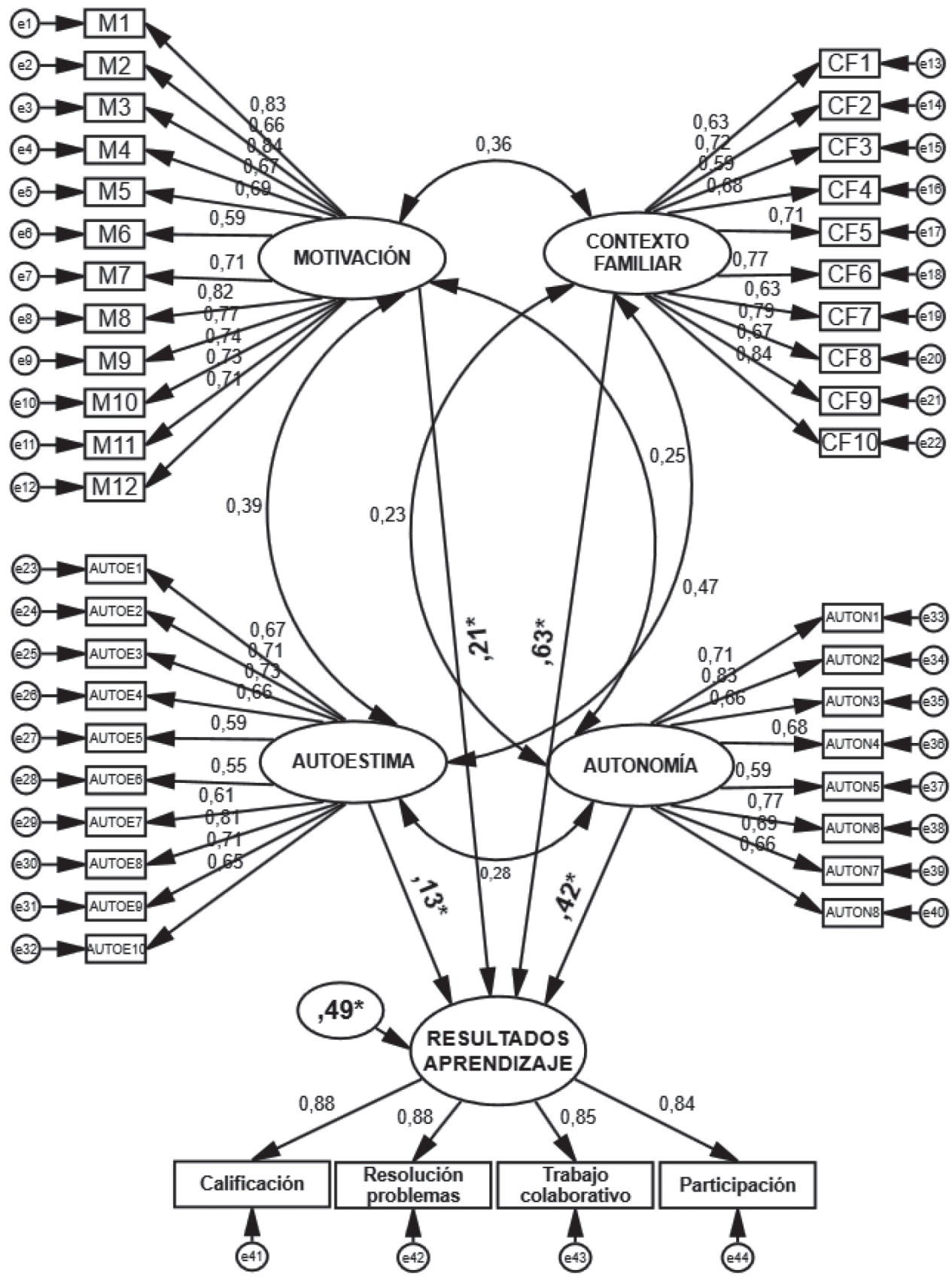

Figura 2. Ecuación estructural predictora de los resultados de aprendizaje

Fuente: Elaboración propia 
Sobre las puntuaciones alcanzadas en los distintos ámbitos estudiados, como se muestra en la tabla 5 , se han obtenido valores centrales en cada uno de los aspectos tenidos en consideración en el estudio, siendo la autonomía del discente la que refleja un ligero distanciamiento a su favor sobre las demás variables.

Tabla 5

Descriptivo de las puntuaciones

\begin{tabular}{lrr}
\hline & Mín.-Máx. & Media (DT) \\
\hline Motivación & $1,92-4,05$ & $2,96(0,41)$ \\
Contexto familiar & $1,8-4,24$ & $2,99(0,44)$ \\
Autoestima & $1,65-4,2$ & $2,97(0,45)$ \\
Autonomía & $1,75-4,5$ & $3,02(0,52)$ \\
Resultados de aprendizaje & $1-5$ & $2,96(0,7)$ \\
\hline
\end{tabular}

Fuente: Elaboración propia

En la Tabla 6 se muestra el resultado del modelo de regresión lineal múltiple realizado para determinar el efecto de las variables demográficas y las variables tomadas como factores externos del flipped learning en los resultados de aprendizaje. El modelo resultante fue estadísticamente significativo $(F(7,599)=5,841 p<0,001)$ y explicó un $52,3 \%$ de la varianza. Las variables sociodemográficas que mostraron un efecto significativo en los resultados de aprendizaje fueron la edad (edades mayores se asocian con mejores resultados) y el hecho de trabajar (trabajar se asocia con bajos resultados). Por otra parte, los factores extrínsecos mostraron un efecto estadísticamente significativo y positivo en los resultados de aprendizaje (niveles altos de contexto familiar, autonomía, motivación y autoestima se asocian con niveles altos en los resultados de aprendizaje). 
SANTIAGO MENGUAL-ANDRÉS, JESÚS LÓPEZ BELMONTE, ARTURO FUENTES CABRERA,

Tabla 6

Efecto de las variables sociodemográficas y los factores extrínsecos sobre los resultados de aprendizaje

\begin{tabular}{llll}
\hline Variable & $\boldsymbol{B}(\boldsymbol{E T})$ & $\boldsymbol{t}$ & $\boldsymbol{p}$-valor \\
\hline Sexo (Mujer) & $0,061(0,041)$ & 1,513 & 0,131 \\
Edad & $0,024(0,009)$ & 2,579 & 0,010 \\
Trabajo (Sí) & $-0,055(0,026)$ & 2,116 & 0,035 \\
Motivación & $0,085(0,038)$ & 2,233 & 0,026 \\
Contexto familiar & $0,537(0,044)$ & 12,074 & $<0,001$ \\
Autoestima & $0,063(0,029)$ & 2,166 & 0,031 \\
Autonomía & $0,139(0,046)$ & 3,041 & 0,002 \\
\hline
\end{tabular}

Fuente: Elaboración propia

\section{DISCUSIÓN Y CONCLUSIONES}

Como ha quedado reflejado en la literatura presentada sobre los estudios de mayor impacto llevados a cabo sobre el flipped learning -como praxis innovadora desarrollada en una era donde está en auge el aprendizaje en entornos digitales- se ha constatado en un análisis de contenido que esta modalidad de enfoque metodológico fomenta la efectividad del aprendizaje (He, et al., 2016; Sánchez, et al., 2019), la motivación de los discentes (Thai, et al., 2017; Tse, et al., 2019), su participación en las acciones formativas (Long, et al., 2016), su capacidad de resolver problemas o situaciones de incertidumbre planteadas por el docente (Bognar, et al., 2019) de manera colaborativa, cooperando el grupo de alumnos para alcanzar los objetivos, tanto en el aula física como en los espacios virtuales (Báez y Clunie, 2019; Kwon \& Woo, 2017; MacLeod, et al., 2017), contribuyendo estos aspectos de manera positiva en la calificación de los estudiantes (Awidi \& Paynter, 2019; Karabulut, et al., 2018; Nortvig, et al., 2018).

De igual manera, los estudios analizados revelan que aspectos como la autonomía -potenciada por este enfoque innovador- (Chaves, et al., 2016; Miño, et al., 2018), la autoestima (Esquivel, 2018) y el contexto familiar (Medranda y Romero, 2018; Tique, et al., 2018) inciden en los resultados alcanzados por los estudiantes en todo proceso formativo.

Concretamente en este estudio, como nuevo hallazgo de la literatura científica sobre los factores determinantes en flipped learning se ha constatado que la variable con mayor índice de influencia sobre los resultados de 
aprendizaje mediante el uso de esta metodología emergente resulta ser el contexto familiar, seguido en menor grado de incidencia por la autonomía, la motivación y la autoestima de los discentes.

Teniendo en cuenta las variables sociodemográficas del estudio, según los valores alcanzados, se ha podido determinar que el sexo de los discentes no influye en la consecución de óptimos resultados de aprendizaje mediante flipped learning. En cambio, la edad y la situación laboral de los estudiantes sí experimentan una incidencia sobre tales resultados en el modelo emergente. Especialmente, se ha comprobado que el alumnado de mayor edad ha obtenido mejores valoraciones y los discentes que disponen de un trabajo mientras cursan sus estudios universitarios provocan un descenso de las mismas.

En cuanto a los factores externos al enfoque flipped learning que se han establecido en esta investigación, se ha obtenido que aquellos participantes que disponen de un contexto familiar adecuado junto con pertinentes valores de autonomía, motivación y autoestima, hayan alcanzado una mayor proyección en los resultados de aprendizaje por medio de esta metodología innovadora mediada por la tecnología.

De este trabajo han derivado un conjunto de prospectivas para mejorar la puesta en marcha del flipped learning en función del contexto familiar del alumnado, por ser uno de los factores que más incidencia ha otorgado sobre el enfoque metodológico en cuestión. Fruto de los hallazgos obtenidos se debe tener en cuenta que para optimizar el proceso de aprendizaje a través de tal metodología tecnológica es necesario contar con un ambiente de trabajo idóneo para la visualización de los contenidos en formato digital, esto es, contar con los recursos tecnológicos necesarios, así como un ambiente y espacio de trabajo adecuado a los requerimientos de una actividad formativa fuera del aula. Es por ello que el docente que esté dispuesto a desplegar un enfoque de aula invertida en sus asignaturas debe tener en cuenta que los resultados de aprendizaje se verán condicionados -mayormente- por las características del contexto familiar de los discentes.

De igual manera, pero en menor escala, la autonomía del alumnado se constituye como otro factor influyente que puede alterar los resultados finales. Por ello, se recomienda que antes del despliegue de una metodología de tal naturaleza, el profesorado realice un trabajo de campo con la finalidad de analizar si su alumnado reúne un perfil autónomo, puesto que de lo contrario la aplicación de una metodología innovadora como flipped learning puede verse afectada y -en consecuencia- no lograr los beneficios y potencialidades que exporta en el aprendizaje. 
Asimismo, en esta línea se encuentran -del mismo modo-la motivación y la autoestima de los estudiantes. A pesar de que han resultado ser los factores con menor grado de incidencia sobre los resultados de aprendizaje alcanzados mediante el uso del flipped learning, como implicación práctica se propone la realización por parte del docente de un feedback con un alto componente psicoeducativo que permita tanto retroalimentar a los discentes sobre su consecución de los objetivos concernientes al proceso de aprendizaje como mejorar su actitud, motivación y autoestima de estos durante la acción formativa. Todo ello por medio de un diálogo constante y fluido que responda a las inquietudes y necesidades del alumnado, asumiendo el docente el rol de coach del aprendizaje. Porque, como dictan los nuevos cánones educativos, el profesorado en la actualidad ya no es un mero transmisor de información y conocimiento sino que pasa a tomar una nueva postura, la de guía del proceso formativo y ahí es donde se encuadra este nuevo rol dinamizador de coaching educativo.

Con todo ello, la decisión del profesorado para aplicar -o no aplicarun enfoque flipped learning puede verse condicionada a estos factores estudiados. No obstante, está en las destrezas del docente el sacar todos los beneficios y potencialidades que una metodología innovadora como esta supone en el proceso de enseñanza y aprendizaje.

La principal limitación hallada en este estudio ha sido la realización periódica de material audiovisual por parte del docente para poder efectuar un proceso de enseñanza y aprendizaje de tales singularidades, así como la abundante carga de trabajo complementario que supuso para el profesional.

Como futura línea de investigación se propone analizar tanto la competencia digital del alumnado como su ocupación familiar, contemplando si desempeña funciones en el contexto familiar como el cuidado de miembros familiares (hijos o personas enfermas) con el propósito de seguir indagando en la influencia sobre los resultados de aprendizaje obtenidos con el modelo flipped learning. 


\section{REFERENCIAS BIBLIOGRÁFICAS}

Awidi, I.T. \& Paynter, M. (2019). The impact of a flipped classroom approach on student learning experience. Computers \& Education, 128, 269-283.

Báez, C.I. \& Clunie, C. E. (2019). Una mirada a la Educación Ubicua. RIED. Revista Iberoamericana de Educación a Distancia, 22(1), 325-344. 10.5944/ ried.22.1.22422

Barbosa, S.H. \& Amariles, M.L. (2019). Learning Styles and the Use of ICT in University Students within a Competency-Based Training Model. Journal of New Approaches in Educational Research, 8(1), 1-6. 10.7821/ naer.2019.1.296

Boelens, R., Voet, M., \& De Wever, B. (2018). The design of blended learning in response to student diversity in higher education: Instructors' views and use of differentiated instruction in blended learning. Computers \& Education, 120, 197-212. 10.1016/j. compedu.2018.02.009

Bognar, B., Sablið, M., \& Škugor, A. (2019). Flipped Learning and Online Discussion in Higher Education Teaching. En C. Reidsema, L. Kavanagh, R. Hadgraft, \& N. Smith (Eds.), The flipped classroom: Practice and practices in higher education (pp. 371-392). Nueva York, USA.: Springer. 10.1007/978-3-030-01551-0_19

Cabanach, R. G., Souto, A., Freire, C. F., y Ferradás, M. M. (2015). Relaciones entre autoestima y estresores percibidos en estudiantes universitarios. European Journal of Education and Psychology, 7(1), 43-57. 10.1989/ejep.v7i1.151

Cabero, J., Arancibia, M.L., \& Del Prete, A. (2019). Technical and Didactic
Knowledge of the Moodle LMS in Higher Education. Beyond Functional Use. Journal of New Approaches in Educational Research, 8(1), 25-33. 10.7821/ naer.2019.1.327

Cabero, J. y Llorente, M. C. (2015). Tecnologías de la Información y la Comunicación (TIC): escenarios formativos y teorías del aprendizaje. Revista Lasallista de Investigación, 12(2), 186-193.

Cendon, E. (2018). Lifelong Learning at Universities: Future Perspectives for Teaching and Learning. Journal of New Approaches in Educational Research, 7(2), 81-87. 10.7821/naer.2018.7.320

Cerezo, R., Bernardo, A., Esteban, M., Sánchez, M., y Tuero, E. (2015). Programas para la promoción de la autorregulación en educación superior: un estudio de la satisfacción diferencial entre metodología presencial y virtual. European Journal of Education and Psychology, 8(1), 3036.

Chaves, E., Trujillo, J. M., y López, J. A. (2016). Acciones para la autorregulación del aprendizaje en entornos personales de aprendizaje. Pixel-bit: revista de medios y educación, 48, 67-82. 10.12795/ pixelbit.2016.i48.05

El Miedany, Y. (2019). Flipped Learning. En C. Reidsema, L. Kavanagh, R. Hadgraft, \& N. Smith (Eds.), The flipped classroom: Practice and practices in higher education (pp. 285303). Nueva York, USA: Springer. 10.1007/978-3-319-98213-7_15

Esquivel, J.C. (2018). Autoestima y su relación con el rendimiento académico en estudiantes del sistema de universidad abierta. Facultad de 
Educación. Universidad Católica los Ángeles de Chimbote-Huaraz, 2015. Aporte Santiaguino, 11(1), 157166. 10.32911/as.2018.v11.n1.464

Froehlich, D.E. (2018). Nontechnological learning environments in a technological world: Flipping comes to the aid. Journal of New Approaches in Educational Research, 7(2), 94-99. 10.7821/naer.2018.7.304

Guri-Rosenblit, S. (2018). E-Teaching in Higher Education: An Essential Prerequisite for E-Learning. Journal of New Approaches in Educational Research, 7(2), 93-97. 10.7821/ naer.2018.7.298

He, W., Holton, A., Farkas, G., \& Warschauer, M. (2016). The effects of flipped instruction on out-of-class study time, exam performance, and student perceptions. Learning and Instruction, 45, 61-71. 10.1016/j. learninstruc.2016.07.001

Hernández, R., Fernández, C., y Baptista, M. P. (2016). Metodología de la investigación. México D.F., México: McGraw Hill.

Hinojo, F.J., Aznar, I., Romero, J.M., y Marín, J.A. (2019). Influencia del aula invertida en el rendimiento académico. Una revisión sistemática. Campus Virtuales, 8(1), 9-18.

Huan, C. (2016). A Study on Digital Media Technology Courses Teaching Based on Flipped Classroom. American Journal of Educational Research, 4(3), 264-267. 10.12691/education-4-3-6

Hwang, G.J. \& Lai, C.L. (2017). Facilitating and Bridging Out-Of-Class and In-Class Learning: An Interactive E-Book-Based Flipped Learning Approach for Math Courses. Journal of Educational Technology \& Society, 20(1), 184-197. Recuperado de https://bit.ly/2W3sNEJ
Jovanović, J., Gašević, D., Dawson, S., Pardo, A., \& Mirriahi, N. (2017). Learning analytics to unveil learning strategies in a flipped classroom. The Internet and Higher Education, 33(4), 74-85. 10.1016/j.iheduc.2017.02.001

Karabulut, A., Jaramillo, N., \& Hassall, L. (2018). Flipping to engage students: Instructor perspectives on flipping large enrolment courses. Australasian Journal of Educational Technology, 34(4), 123-137. 10.14742/ ajet.4036

Kwon, J.E. \& Woo, H.R. (2017). The Impact of Flipped Learning on Cooperative and Competitive Mindsets. Sustainability, 10(79), 1-15. 10.3390/su10010079

Lee, J., Lim, C., \& Kim, H. (2017). Development of an instructional design model for flipped learning in higher education. Educational Technology Research and Development, 65(2), 427453. 10.1007/s11423-016-9502-1

Lee, J., Park, T., \& Davis, R. O. (2018). What affects learner engagement in flipped learning and what predicts its outcomes?. British Journal of Educational Technology. 1(1), 1-18. 10.1111/bjet.12717

Long, T., Cummins, J., \& Waugh, M. (2017). Use of the flipped classroom instructional model in higher education: instructors' perspectives. Journal of Computing in Higher Education, 29(2), 179-200. 10.1007/s12528-016-9119-8

Long, T., Logan, J., \& Waugh, M. (2016). Students' perceptions of the value of using videos as a pre-class learning experience in the flipped classroom. TechTrends, 60(3), 245-252. 10.1007/s11528-016-0045-4

López, M. y Bernal, C. (2019). El perfil del profesorado en la Sociedad Red: 
SANTIAGO MENGUAL-ANDRÉS, JESÚS LÓPEZ BELMONTE, ARTURO FUENTES CABRERA, SANTIAGO POZO SÁNCHEZ

MODELO ESTRUCTURAL DE FACTORES EXTRÍNSECOS INFLUYENTES EN EL FLIPPED LEARNING

reflexiones sobre la competencia digital de los y las estudiantes en Educación de la Universidad de Cádiz. IJERI: International Journal of Educational Research and Innovation, (11), 83-100. Recuperado de https://bit. ly/2LIr8F0

MacLeod, J., Yang, H.H., Zhu, S., \& Shi, Y. (2017). Technological factors and student-to-student connected classroom climate in cloud classrooms. Journal of Educational Computing Research, 56(6), 826-847. doi: 10.1177/0735633117733999

McCarthy, J. (2016). Reflections on a flipped classroom in first year higher education. Issues in Educational Research, 26(2), 332-350. Recuperado de https://bit.ly/2WeppvH

Medranda, R.F. y Romero, S. (2018). Problemas familiares y rendimiento académico. Revista Caribeña de Ciencias Sociales, 4(2), 1-11. Recuperado de https://bit.ly/2K9XWEt

Nortvig, A.M., Petersen, A.K., \& Hattesen, S. (2018). A Literature Review of the Factors Influencing E-Learning and Blended Learning in Relation to Learning Outcome, Student Satisfaction and Engagement. Electronic Journal of e-Learning, 16(1), 46-55. Recuperado de https://bit.ly/2W4iMHL

Pereira, S., Fillol, J. y Moura, P. (2019). El aprendizaje de los jóvenes con medios digitales fuera de la escuela: De lo informal a lo formal. Comunicar: Revista científica iberoamericana de comunicación y educación, 27(58), 4150. 10.3916/C58-2019-04

Rotellar, C. \& Cain, J. (2016). Research, perspectives, and recommendations on implementing the flipped classroom. American journal of pharmaceutical education, 80(2), 1-9. 10.5688/ajpe80234

Sánchez, C. (2017). Flipped classroom. La clase invertida, una realidad en la Facultad de Ciencias de la Educación de la Universidad de Málaga. [Tesis doctoral]. Universidad de Málaga. Recuperado de https://bit.ly/2XgaFZJ

Sánchez, J L., Jimeno, A., Pertegal, M.L., \& Mora, H. (2019). Design and application of Project-based Learning Methodologies for small groups within Computer Fundamentals subjects. IEEE Access, 7, 12456-12466. 10.1109/ACCESS.2019.2893972

Thai, N.T T., De Wever, B., \& Valcke, M. (2017). The impact of a flipped classroom design on learning performance in higher education: Looking for the best "blend" of lectures and guiding questions with feedback. Computers \& Education, 107, 113-126. 10.1016/j. compedu.2017.01.003

Tique, M.A., Camacho, J.A., Segura, Y.C., Orozco, R., Ortega, L.L., Iriarte, M. ... Cáceres, N.A. (2018). Contexto socioeconómico sobre el rendimiento académico del estudiante mediante la investigación como estrategia pedagógica. Cultura Educación y Sociedad, 9(1), 171-180. 10.17981/ cultedusoc.9.1.2018.13

Tourón, J. y Santiago, R. (2015). El modelo Flipped Learning y el desarrollo del talento en la escuela. Revista de educación, 368, 174-195. 10.4438/1988-592X-RE-2015368-288

Tse, W.S., Choi, L.Y., \& Tang, W.S. (2019). Effects of video-based flipped class instruction on subject reading motivation. British Journal of Educational Technology, 50(1), 385398. 10.1111/bjet.12569 
Yilmaz, R. (2017). Exploring the role of e-learning readiness on student satisfaction and motivation in flipped classroom. Computers in Human Behavior, 70, 251-260. 10.1016/j. chb.2016.12.085
Zainuddin, Z., Habiburrahim, H., Muluk, S. \& Keumala, C.M. (2019). How do students become self-directed learners in the EFL flipped-class pedagogy? A study in higher education. Indonesian Journal of Applied Linguistics, 8(3). 10.17509/ijal.v8i3.15270 


\title{
PERFIL ACADÉMICO Y PROFESIONAL DE LOS AUTORES
}

Santiago Mengual-Andrés. Doctor en Investigación Educativa por la Universidad de Alicante. Profesor Contratado Doctor del Departamento de Educación Comparada e Historia de la Educación de la Facultad de Filosofía y Ciencias de la Educación de la Universidad de Valencia. Actualmente desempeña su investigación como miembro del grupo de investigación de Políticas Educativas, Interculturalidad y Sociedad (POLISOC).

Jesús López Belmonte. Doctor en Ciencias de la Educación, con Máster Universitario en tecnología educativa y competencias digitales. Es miembro del grupo de investigación AREA (HUM-672) de la Universidad de Granada. Actualmente desempeña su labor docente en la Universidad Internacional de Valencia y en el Centro Concertado Beatriz de Silva (Ceuta).

Arturo Fuentes Cabrera. Doctor en Ciencias de la Educación. Profesor de la Facultad de Educación, Economía y Tecnología de Ceuta y Profesor en la Universidad Nacional de Educación a Distancia (UNED). Es miembro del grupo de investigación AREA (HUM-672) de la Universidad de Granada. Actualmente desarrolla su profesión docente en el Departamento de Métodos de Investigación y Diagnóstico en Educación de la Universidad de Granada.

Santiago Pozo Sánchez. Doctorando en Ciencias de la Educación por la Universidad de Granada, en el Departamento de Didáctica y Organización Escolar. Posee un Máster Universitario en Profesorado de Educación Secundaria. Es miembro del grupo de investigación AREA (HUM-672) de la Universidad de Granada. Actualmente compagina su labor investigadora con la docencia en el Centro Concertado Beatriz de Silva (Ceuta).

\author{
Dirección de los autores: Santiago Mengual-Andrés \\ Universidad de Valencia \\ Departamento de Educación Comparada e \\ Historia de la Educación \\ Facultad de Filosofía y Ciencias de la \\ Educación \\ Avda. Blasco Ibáñez 13, \\ 46010, Valencia (España) \\ E-mail: santiago.mengual@uv.es \\ Jesús López Belmonte \\ Universidad Internacional de Valencia \\ Departamento de Educación \\ C/ Pintor Sorolla 21,
}


SANTIAGO MENGUAL-ANDRÉS, JESÚS LÓPEZ BELMONTE, ARTURO FUENTES CABRERA,

46002, Valencia (España)

E-mail: jesus.lopezb@campusviu.es

Arturo Fuentes Cabrera

Universidad de Granada

Departamento de Métodos de Investigación

y Diagnóstico en Educación

Facultad de Educación, Economía y

Tecnología de Ceuta

C/ Cortadura del Valle s.n,

51001, Ceuta (España)

E-mail: arturofuentes@ugr.es

Santiago Pozo Sánchez

Universidad de Granada

Departamento de Didáctica y Organización

Escolar

C/ Cortadura del Valle s.n,

51001, Ceuta (España)

E-mail:santiagopozo@correo.ugr.es

Fecha Recepción del Artículo: 14. Febrero. 2019

Fecha Modificación del Artículo: 06. Junio. 2019

Fecha Aceptación del Artículo: 10. Junio. 2019

Fecha Revisión para Publicación: 19. Junio. 2019 
